\title{
Lung Cancer: Future Challenges and Opportunities
}

\section{Sandra Hodge*}

Department of Thoracic Medicine, Lung Research, Royal Adelaide Hospital, Adelaide, Australia

Over 1.5 million people worldwide die from lung cancer per year. The disease has an extremely poor prognosis and there has been no improvement in the 5 -year survival rate in the last 30 years. Unfortunately currently available treatments do not prevent the relentless progression of the disease. There has been lack of optimism regarding treatment advances in the last ten years, and progress in treating or predicting the risk for lung cancer has been slow. As highlighted in the article by Lamelas et al. [1] in this issue, ongoing efforts to improve the survival of patients with lung cancer should be centered on achieving an earlier diagnosis in addition to developing new targeted or individualized therapies for the disease.

Patients are currently diagnosed with lung cancer when their disease is fairly advanced, as this is when they begin to experience symptoms. Sadly, at this stage, surgery or radiotherapy are usually not viable options, and chemotherapy is usually commenced. The importance of accurate prediction of lung cancer at an early stage is therefore of utmost importance, and Gaber et al. [2] presents herein a comprehensive assessment of the problems that may impact a diagnosis of lung cancer during the first visit to a fast track clinic, and provides useful data to advise early diagnostic accuracy.

In this issue of PRM we are provided with an exciting and timely update on emerging diagnostic or prognostic biomarkers and therapeutic approaches for lung cancer. Recent approaches include novel targeting of angiogenic factors including Vascular Endothelial Growth Factor (VEGF) and basic Fibroblast Growth Factor (bFGF) produced by Non-Small Cell Lung Carcinoma (NSCLC). VEGF is known to promote the vascularization and growth of the tumor by mobilizing circulating endothelial cell precursors to the nascent blood vessels and plays a key role in establishing new metastatic foci. bFGF in addition to its involvement in tumor growth, may have the potential to predict early-stage NSLC recurrence following tumor resection. Ghany et al. [3] in the article that accompanies this commentary, report that Nimesulide, an antiangiogenic selective COX-2 inhibitor, showed promising results as an add-on therapy with traditional chemotherapy. Levels of pro-angiogenic parameters were normalized, potentially having a positive effect on the outcome and prolongation of survival rate in patients with NSLC.

VEGF and bFGF were sensitive markers of NSLC patients' prognosis. Studies such as these that provide us with a better understanding of the molecular origins and development of lung cancer are important if we are to improve the survival and life quality of patients. Also discussed by De Mello in this issue [4], are recent advances in understanding the genetic influence on lung cancer risk. These advances are gaining impetus and are likely to provide more effective therapies and predictive biomarkers in the near future. We know that some patients who do not respond to therapy have underlying genetic faults driving their cancer. These patients are therefore good candidates for new, targeted therapies based on the specific genetic fault. Huge success in this line of treatment has been already demonstrated with EGFR-targeted therapies (eg, cetuximab and erlotinib).

Other potential targeted therapies are under investigation including BRAF, HER-2, c-KIT and EML4-ALK.Emerging genetic markers of lung cancer risk have also been reported. From the numerous studies that have emerged over the last 10 years, a Single Nucleotide Polymorphism (SNP) at chromosome $13 \mathrm{q} 31.3$, and $\mathrm{EGF}=61 \mathrm{~A}>\mathrm{G}$ polymorphisms have emerged as promising markers of NSLC risk, but further studies are required. To offer the most effective targeted therapy, it is now essential to sub-classify NSLC and perform molecular analyses. As presented by Pusztaszeri et al. [5] in this edition, recent advances in rapid on-site evaluation and novel endoscopic techniques are facilitating cytological diagnoses.

Taken together, the data and views presented in this issue of PRM thus provide us with encouraging evidence that we are finally making progress in better understanding the pathogenesis of lung cancer and the genetic background of metastasis, and that we are getting closer to developing the next generation of more effective targeted therapies. However we still have a long journey ahead, especially to tackle the development of resistance to NSLC treatment, and one of our main challenges in the future is to facilitate efficient and personalized therapeutic management of lung cancer.

\section{References:}

1. Lamelas IP, Arca JA, Pérez MTA, García MJG, Velázquez PM (2012) Long-Term Lung Cancer Survival in a Province of Spain. J Pulm Respir Med S5: 001.

2. Gaber KA, DeBarr SE, Stevens S, Elkkari A, Bakere H, et al. (2012) Ability of Lung Specialist to Predict a Diagnosis of Lung Cancer during the First Visit to a Fast Track Clinic. J Pulm Respir Med S5: 004.

3. Abdel Ghany SM, El Melegy NT, Mohamed-Hussein AAR, Hana RS (2011) Emerging Prognostic Biomarkers in Non Small Cell Lung Cancer Patients: Impact of Treatment with Nimesulide (COX-2 Inhibitor) Combined with Chemotherapy. J Pulm Respir Med S5:003.

4. Mello RAD (2012) EGF+61 A>G Polymorphisms and Lung Cancer Risk: Future Directions. J Pulm Respir Med S5: e001.

5. Pusztaszeri M, Soccal PM, Mach N, Pache J, Mc Kee T (2012) Cytopathological Diagnosis of Non Small Cell Lung Cancer: Recent Advances Including Rapid OnSite Evaluation, Novel Endoscopic Techniques and Molecular Tests. J Pulm Respir Med S5: 002.

*Corresponding author: Sandra Hodge, Department of Thoracic Medicine, Lung Research, Royal Adelaide Hospital, Adelaide, Australia, E-mail: Sandra.Hodge@ health.sa.gov.au

Received October 11, 2013; Accepted October 14, 2013; Published October 16 2013

Citation: Hodge S (2013) Lung Cancer: Future Challenges and Opportunities. J Pulmon Resp Med S5: 005. doi: 10.4172/2161-105X.S5-005

Copyright: () 2013 Hodge S. This is an open-access article distributed under the terms of the Creative Commons Attribution License, which permits unrestricted use, distribution, and reproduction in any medium, provided the original author and source are credited. 